\title{
CLASSIFICAÇÃO INTERNACIONAL DAS ATIVIDADES DE ENFERMAGEM: UMA AMOSTRA DA REALIDADE BRASILEIRA*
}

\author{
Magda Rojas Yoshioca** \\ Maria Alves Barbosa** \\ Nádia Zanon Narchi*** \\ Isabel Cristina F. da Cruz** \\ Ana Lucia Queiroz Bezerra*** \\ Rose Meire Imanichi***
}

\begin{abstract}
RESUMO: O estudo constituiu-se de levantamento bibliográfico com objetivo de identificar os termos referentes às atividades de enfermagem: diagnosticar, prescrever e avaliar. O levantamento foi realizado em revistas, dissertações e teses da enfermagem brasileira, do acervo da biblioteca da Escola de Enfermagem da Universidade de São Paulo e do catálogo de pesquisas e pesquisadores de enfermagem do Centro de Estudos e Pesquisas da Associação Brasileira de Enfermagem, no período de 1988 a 1992. Das 119 publicações encontradas identificou-se que $53,5 \%$ referiam-se ao termo prescrição de enfermagem, 39,0\% ao diagnóstico de enfermagem e $7,5 \%$ à avaliação da assistência. Cada um destes termos recebeu denominações diferentes, o que denota a necessidade de padronização de linguagem.
\end{abstract}

ABSTRACT: The study was compounded by bibliography collecting with the objective of identifying terms referred to nursing activities: to diagnose, prescribe and evaluate. The data collecting was done by making use of Brazilian nursing journals, dissertations and the theses from nursing school's heap of Brazilian library belonging to Universidade de São Paulo. Catalogues and nursing researches from the Research and Study Center from Brazilian Nursing Association were used, for the period of time between 1988 and 1992. From 119 issues which were found it was shown that 53,5\% referred to a nursing prescription term, $39,0 \%$ was related to nursing diagnostic and $7,5 \%$ to assistance evaluation. Each of these terms got different denominations which show the patterned language necessity.

\section{INTRODUÇÃO}

O propósito deste trabalho é contribuir nas discussões sobre a padronização da linguagem ref erente às atividades de enfermagem: diagnóstico, prescrição e avaliação do resultado da assistência. Nesse sentido KIM+, presidente do ICN (International Council of Nursing), considera que deva existir relação entre nomenclatura, linguagem, classificação e padrões mínimos de assistência, para que se implemente um sistema voltado para a prática e que ao mesmo tempo garanta a sua retroalimentação.

O ICN sugere também, que os critérios para a elaboração da classificação da prática de enfermagem, sejam os de atender aos múltiplos propósitos dos diferentes países, ter linguagem acessível e adequada, possuir uma definição conceitual que independa do

\footnotetext{
* Trabalho apresentado no $45^{\circ}$ Congresso Brasileiro de Enfermagem. Recife - PE, 28 de novembro a 3 de dezembro de 1993.

** Doutorandas da Escola de Enfermagem da Universidade de São Paulo.

*** Mestrandas da Escola de Enfermagem da Universidade de São Paulo

$+\quad$ KIM (International Council of Nursing). Pesquisa e assistência: panorama e tendências mundiais. [ Conferência proferida durante o Encontro Internacional "Pesquisa em Enfermagem: uma questão de saúde", São Paulo, 1992]
} 
referencial teórico e do modelo de assistência, conter núcleo central ao qual se permita um processo contínuo de desenvolvimento e aperfeiçoamento, ser sensível à variedade cultural, refletir sistemas de valores comuns de enfermagem, como aqueles contidos no seu código para enfermeiros, e ser adotado pela Organização Mundial da Saúde (OMS) para inclusão ou complementação do Código Internacional de Doenças $(\mathrm{CID})^{(35)}$.

A classificação das atividades de enfermagem, para ser sistematizada e universalmente aceita, necessita que os elementos básicos - diagnóstico, prescrição e avaliação de enfermagem - sejam claramente explicitados $(5,10,32,33)$.

Os primeiros trabalhos que mostram a preocupação com a universalização de linguagem para a prática da enfermagem, surgiram na década de setenta entre enfermeiros americanos e canadenses, que propuseram a criação de modelos para classificação internacional de diagnósticos de enfermagem. Eles diferem entre si na concepção de abordagem da clientela, mas têm como ponto comum o estabelecimento de uma cientificidade que possa estar contida na praxis dos enfermeiros internacionalmente considerados.

Nesse sentido também existe a tentativa de enfermeiros americanos padronizarem prescrições de enfermagem baseadas na classificação de diagnósti$\cos ^{(32,33)}$.

No Brasil existem pesquisadores preocupados em estudar diagnósticos de enf ermagem (11, 12, 13, 14. $15,16,18,20,31,34,38)$. Esses estudos constituem os primeiros passos em direção à uniformização de linguagem no país, e deverão servir de subsídio para a elaboração de classificação internacional da prática de enfermagem contendo os elementos básicos - diagnóstico, prescrição e avaliação.

O presentc estudo tem por objetivo identificar a produção de trabalhos da enfermagem brasileira quanto às atividades caracterizadas pelos itens: diagnóstico, prescrição e avaliação de enfermagem através de levantamento bibliográfico do período de 1988 a 1992.

\section{METODOLOGIA}

Foram consultadas revistas, dissertações e teses de enfermagem do acervo da Escola de Enfermagem da Universidade de São Paulo (EEUSP), e do catálogo sobre pesquisas e pesquisadores do Centro de Estudos c Pesquisa em Enfermagem da Associação Brasilcira de Enfermagem (ABEn).

Dessas referências, extraíram-se 119 publicações que constituem a população deste trabalho. A análise dos textos apontou para a necessidade de se agrupar os termos, segundo as semelhanças e o sentido, em diagnóstico, prescrição e avaliação. Os termos assim identificados aparecem isoladamente ou em conjunto nos textos, sendo sua análise realizada de forma separada conforme o agnupamento.

Neste estudo, entende-se por diagnóstico o julgamento clínico das respostas do indivíduo, família ou comunidade ao problema de saúde ou processo vital.

Por prescrição, compreende-se que são as intervenções realizadas ou delegadas pelo enfermeiro, para resolução do diagnóstico de enfermagem ou não, tendo em vista o resultado a ser alcançado.

$O$ resultado proveniente das atividades do enfermeiro, dirigidas para a resolução de um determinado diagnóstico de enfermagem, constitui-se na avaliação.

\section{DISCUSSÃO DOS RESULTADOS}

A Figura 1, a seguir, demonstra a totalidade das atividades de enfermagem - diagnóstico, prescrição e avaliação - no âmbito deste trabalho.

Figura 1: Distribuição dos termos identificados COMO DIAGNÓSTICO, PRESCRIÇÃO e AVALIAÇÃO NOS trabalhos referentes às atividades de engermagem, 1988/1992. São Paulo, 1992.

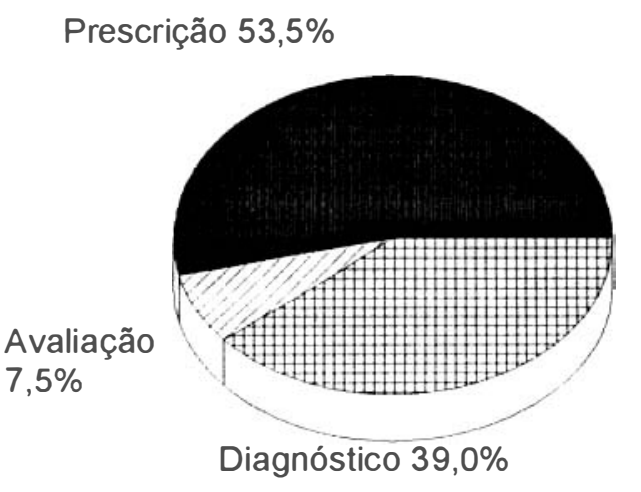

Nesta figura, a prescrição de enfermagem aparece com maior freqüência $(53,5 \%)$. Supõe-se que isto se deva à privativa do enf crmeiro e previstas na Lei do Exercício Profissional de Enfermagem ${ }^{(3,4)}$.

Quanto ao diagnóstico de enfermagem, verificase freqüência de $7,5 \%$. o que sugere ser esta uma área 
incipiente de pesquisa.

A seguir, os dados serão apresentados conforme agnupamento dos termos: diagnóstico, prescrição $e$ avaliação.

\subsection{Diagnóstico de Enfermagem}

O termo diagnóstico tem origem grega e significa conclusão ou julgamento resultante de um processo analítico.

Segundo HORTA ${ }^{(27)}$ o diagnóstico constitui um dos elementos do processo de enfermagem, sendo definido como: “ "... a identificação das necessidades básicas do indivíduo (família ou comunidade) que precisam de atendimento e a determinação, pela Enfermagem, do grau de dependência deste atendimento em natureza a extensão".

PAIM et al. (36) definem diagnóstico de enfermagem como "a determinação da dependência que o paciente tem do enfermeiro em termos de necessidades humanas afetadas e a partir dos problemas de enfermagem extraídos do histórico de enfermagem do paciente".

CAMPEDELLI et al. ${ }^{(8)}$ consideram como diagnóstico de enfermagem as impressões do examinador (o enfermeiro) sobre seu cliente. A impressão do examinador confere caráter subjetivo ao diagnóstico, enquanto que a classificação sobre a terminologia, para o diagnóstico, garante objetividade para a prática de enfermagem.

No conjunto das publicações sobre o tema, percebe-se na obra de HORTA(27) a busca pela especificação da essência do diagnóstico. Nota-se preocupação da autora quanto a necessidade de existir visão de conjunto dos dados coletados no histórico e de nomeá-los, ou seja, fazer diagnóstico. A referida autora propôs então, as Sindromes de Enfermagem. compreendidas como forma embrionária de se nomear as respostas do cliente ao seu problema de saúde ou processo vital.

Na literatura brasilcira consultada, vê-se que a sistematização das atividades de enfermagem iniciase a partir da década de 50 com as pesquisas de

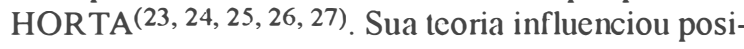
tivamente a enfermagem brasileira, pois a partir de seu trabalho, os profissionais começaram a atuar de forma sistematizada. $O$ não ajustamento às dificuldades iniciais existentes, quando se implementou a nova metodologia, assim como o não aprofundamento ou aperfeiçoamento pelos usuários de suas propostas, constituem limitações na utilização dessa teoria.

Embora o modelo de sistematização da assistência de HORTA seja adotado por algumas escolas e serviços de enfermagem, constata-se por meio da literatura profissional, que a implementação do modelo não observa, por exemplo, a correlação entre as atividades: histórico - diagnóstico - prescrição - avaliação. Desse modo, algumas das etapas do processo de enfermagem são suprimidas, por serem consideradas complexas. $O$ diagnóstico de enfermagem não é implementado, sendo substituído por sinais, sintomas, equipamentos, procedimentos e diagnósticos médicos ${ }^{(8,17,30)}$.

Enquanto atividade profissional, o diagnóstico de enfermagem não está contemplado de forma explícita na legislação do exercício de enfermagem ${ }^{(3,4)}$. Todavia, a consulta de enfermagem deve compreender as etapas do levantamento de dados, diagnóstico, prescrição e avaliação da assistência prestada.

A partir da segunda metade da década de 80,0 conceito de diagnóstico tem ressurgido entre os enfermeiros brasileiros com um novo enfoque. Na atual fase, esse conceito aproxima-se do que foi operacionalmente definido pela North American Nursing Diagnosis Association (NANDA) como "um julgamento clínico das respostas do indivíduo, da família ou da comunidade aos processos vitais ou aos problemas de saúde atuais ou potenciais, os quais fornecem a base para a seleção das prescrições de enfermagem, e para avaliação da assistência pela qual o enfermeiro é reponsável"(39).

A classificação dos diagnósticos de enfermagem proposta pela NANDA começa a ser utilizada, no Brasil, nas instituições assistenciais de ensino em São Paulo, Rio de Janeiro e Paraíba.

Na Paraíba, organizou-se o primeiro Grupo de Interesse em Diagnóstico de Enfermagem (GIDEPB) e em São Paulo o segundo Grupo (GIDE-SP), ambos vinculados à Associação Brasileira de Enfermagem (ABEn). Dos trabalhos desses grupos resultaram até o momento dois Simpósios Nacionais sobre Diagnóstico de Enfermagem (São Paulo, 1991 e Paraíba, 1992).

Durante o $42^{\circ}$ Congresso Brasileiro de Enfermagem, em 1990, o GIDE-PB recomendou a criação de um Fórum Nacional sobre Diagnóstico de Enfermagem. Em 1991, foi criado o Comitê Nacional de Revisão dos Diagnósticos de Enfermagem, para coordenar no Brasil os estudos relativos à tradução da Taxonomia I da NANDA. Ressalte-se que vários 
trabalhos sobre o tema têm sido apresentados em eventos nacionais e internacionais.

A utilização de diagnósticos de enfermagem no Brasil não é absolutamente uniforme, pois encontrase em algumas instituições o desenvolvimento de atividades de enfermagem sistematizadas, incluindo aí o diagnóstico de enfermagem segundo a Taxonomia I da NANDA e. em outras, a ausência de tais atividades.

A expressão "diagnóstico de enfermagem" não é a única utilizada para se referir às respostas do cliente ao problema de saúde. Esta afirmação pode ser claramente demonstrada através do que consta na Tabela 1. Nota-se nesta Tabela que o termo diagnóstico foi o mais empregado $(34,2 \%)$ nos trabalhos, sendo seguido por problemas $(28,8 \%)$.

Analisando-se os estudos que empregam os termos identificados como diagnóstico, pode-se constatar que na maioria das vezes, eles não se constituiam em parte integrante do processo de assistência sistematizada de enfermagem e, algumas vezes, foram utilizados juntamente com os outros elementos identificados como prescrião $e$ avaliação, também de forma não sistematizada.

Tabela 1 - Terminologia identificada como DIAGNÓSTICO em trabalhos referentes às atividades de enfermagem - 1988/1992. São Paulo, 1992.

\begin{tabular}{l|r|r}
\hline TERMO & \multicolumn{1}{c}{ F } \\
\hline Diagnóstico & 25 & 34,2 \\
Problema & 21 & 28,8 \\
Avaliação & 8 & 10,9 \\
Alteração & 7 & 9,6 \\
Paråmetro & 2 & 2,7 \\
Classificação & 2 & 2,7 \\
Manifestação & 2 & 2,7 \\
Indicador de desequilíbrio & 1 & 1,4 \\
Limitaçăo & 1 & 1,4 \\
Comportamento esperado & 1 & 1,4 \\
Impressões do examinador & 1 & 1,4 \\
Taxonomia das funções & 1 & 1,4 \\
Diagnóstico médico & 1 & 1,4 \\
\hline TOTAL & 73 & 100,0 \\
\hline
\end{tabular}

O termo problema, identificado 21 vezes na produção científica consultada, foi aplicado, na maioria das vezes, como problema do paciente segundo patologia ou situação à qual estava exposto. Isso pode ser parcialmente explicado, analisando-se a definição de diagnóstico de enfermagem enunciado por $\operatorname{HORTA}^{(27)}$, onde ele está identificado como problema de enfermagem.

O problema do paciente e o problema de enfermagem são termos que estão intimamente relacionados e muitas vezes confundidos ${ }^{(21)}$. Observou-se neste estudo que o termo problema foi empregado para designar problema do paciente.

Os outros termos encontrados com menor frequência (avaliação, alteração, parâmetro, classificação, manifestação, indicador de desequilíbrio, limitação, comportamento esperado, impressões do examinador, taxonomia das funções, diagnóstico médico), mostram a diversidade de nomenclatura empregada para designar uma mesma atividade de enfermagem - diagnosticar.

Supõe-se que os pesquisadores empregam alguns termos (parâmetros, indicadores de desequilíbrio e taxonomia das funções), movidos pelas tendências do momento da realização da pesquisa e utilizando terminologias emergentes.

Em apenas um trabalho o enfermeiro admitiu que o seu diagnóstico era baseado no diagnóstico médico. Sabe-se porém que, pautados no modelo bio-médico, os enfermeiros, em geral, têm assumido ou adotado como parâmetro para seu diagnóstico, o diagnóstico de outro profissional, em particular, o do médico.

Constatou-se que a terminologia identificada como diagnóstico de enfermagem aparece em diversas especialidades, tais como: saúde materno-infantil (25), saúde do adulto (18), saúde do idoso (3), saúde da família (2), saúde mental (1), práticas alternativas (1), c outras em que não foi possível identificar a que especialidade pertenciam (11).

A diversidade de terminologias empregadas nos trabalhos analisad̦os, para designar diagnóstico de enfermagem, demonstra a dificuldade e a conseqüente necessidade de padronização de linguagem.

\subsection{Prescrição de Enfermagem}

A partir dos trabalhos de HORTA outros foram surgindo c. desse modo, cada parte do processo de enfermagem vem sendo estudada detalhadamente ${ }^{(6,}$ $7,13,14,15,16.17,18,19,20,22,31,34,37,38$ ).

A prescrição de enfermagem é considerada como a tomada de decisão. para indicar qual o tipo de cuidado que deve ser prestado numa determinada 
situação. Isto requer do enfermeiro experiência e capacidade de escolha entre uma ou outra prescrição a ser realizada. ${ }^{(37)}$

Prescução de enfermagem é entendida também como a implementação do plano assistencial pelo roteiro diário, que coordena a ação da equipe de enfermagem, na execução dos cuidados adequados ao atendimento das necessidades básicas e específicas do ser humano(27); ou, ainda, como o conjunto de condutas decididas pela enfermeira, que direciona e coordena a assistência de enfermagem ao paciente de forma individualizada e contínua ${ }^{(1)}$.

É importante comentar que o termo prescrição de enfermagem, inicialmente proposto por diversos autores e reconhecido pela Lei do Exercício Profissional na década de 80 , ganha uma nova sinonímia intervenção - caracterizada pcla definição de GUTIERREZ ${ }^{(22)}$. A autora afirma que a intervenção se dá quando se reconhecem as limitações que o ser humano tem no atendimento de suas necessidades básicas que, apesar de terem caráter universal, variam quanto à sua natureza para o bem estar individual, em decorrência de múltiplos fatores, como os biológicos, os psicológicos e os sociais.

A terminologia identificada como prescrição de enfermagem encontra-se na Tabela 2. Esta tabcla mostra que as mais utilizadas foram: ações (14\%), orientação (13\%), ações educativas ( $11 \%)$, intervenção $(10 \%)$, cuidados $(8 \%)$, plano $(7 \%)$, condutas $(6 \%)$, atividades $(5 \%)$, assistência $(4 \%)$ c programa de orientação $(4 \%)$.

No Brasil, adotou-se legalmente o termo prescrição de enfermagem para caracterizar as intervenções em concordância com a Lei do Exercício Profissional, a qual garante o direito ao enfermeiro de exccutá-la ${ }^{(3,4)}$ Apesar disso, no presente cstudo, em apenas três dos artigos analisados, os autores denominam prescrições às intervenções realizadas ou delegadas pclo enf crmciro para a resolução do diagnóstico de enfermagem.

A análise paralcla do matcrial estudado permite afirmar que as prescrições, assim como outras partes do processo de enfermagem, estiveram ao longo da história da assistência, baseadas no diagnóstico c prescrição médicas, retratando com fidedignidade o modelo bio-médico, firmemente ligado ao pensamento cartesiano.
Tabela 2 - Terminologia identificada como PRESCRIÇÃO, em trabalhos referentes às atividades de enfermagem - 1988/1992. São Paulo, 1992.

\begin{tabular}{l|c|c}
\hline TERMO & \multicolumn{1}{c}{ F } & \% \\
\hline Açס̃es & 14 & 14 \\
Orientaçăo & 13 & 13 \\
Ação educativa & 11 & 11 \\
Intervençăo & 10 & 10 \\
Cuidados & 08 & 08 \\
Plano* & 07 & 07 \\
Condutas & 06 & 06 \\
Atividades & 05 & 05 \\
Assistência & 04 & 04 \\
Programa de orientaçăo & 04 & 04 \\
Prescriçăo & 03 & 03 \\
Procedimentos & 03 & 03 \\
Programa educativo & 03 & 03 \\
Atuaçăo & 02 & 02 \\
Procedimento sistematizado & 02 & 02 \\
de assistência & & \\
Medidas gerais de assistência & 02 & 02 \\
Planejamento da assistência & 01 & 01 \\
Terapêutica de enfermagem & 01 & 01 \\
Tarefa & 01 & 01 \\
Medida terapêutica & 01 & 01 \\
Auto-cuidado & 01 & 01 \\
\hline TOTAL & 100 & 100 \\
* Como Plano foram agrupados plano assistencial, plano de \\
orientaçăo, plano de cuidados e plano terapêutico. \\
$\quad$
\end{tabular}

Outra constatação deste estudo, foi a existência de prescrições de enfermagem fora do modelo alopático de assistência. o que de certa forma evidencia prenúncios de mudanças quanto às atitudes em relação ao fenômeno saúde-doença, representando "um caminho para transcender os limites impostos pelo paradigma dominante' ${ }^{\prime}(29)$

A terminologia identificada como prescrição de enfermagem aparece nos trabalhos das áreas de saúdd matcrno-infantil (31) e na de saúde do adulto (31), surgindo $\mathrm{em}$ menor frequência nas de saúde do idoso (3). saúde mental (3), práticas alternativas (3), saúde da família (2), saúde do trabalhador (1) e em outras em que não foi possível identificar as áreas de especialidades a que pertenciam ${ }^{(14)}$.

A análise dos textos utilizados neste trabalho permitiu identificar diversidade de tcrminologia empregada pelos enfermeiros quando se ref $\mathrm{crem}$ às prescrições de enfermagem. Este fato não permite uso de linguagem comum entre os profissionais, dificultando a padronização de condutas. 


\subsection{Avaliação de Resultados da Assistência de Enfermagem}

A avaliação constitui-se no resultado de processo de trabalho previamente estabelecido através de critérios ou objetivos da assistência de enfermagem.

$O$ processo de trabalho de enfermagem, quer no modelo individual ou coletivo, compreende o objeto natural (clientela), o objeto do trabalho (corpos individuais), o processo propriamente dito (seus agentes e instrumentos) e o produto que é o resultado do processo de trabalho da enfermagem; a avaliação é que norteia a reformulação do processo.

A avaliação, como uma das fases da assistência de enfermagem, serve de "feedback" para reformulação e ajuste do planejamento da assistência e da prescrição de enfermagem. ${ }^{(28)}$

Segundo recomendação do Manual de Padrões Mínimos de Assistência de Enfermagem em Recuperação da Saúde, do Ministério da Saúde, a avaliação é a análise quantitativa e qualitativa da assistência prestada ao paciente, de acordo com objetivos, metas, normas e padrões pré-estabelecidos( ${ }^{(2)}$. O Manual contém critérios de avaliação que abrangem dados específicos referentes a diagnóstico.

O primeiro trabalho de enfermagem no Brasil, que aborda a avaliação dos resultados da assistência de enfermagem, foi o de HORTA(27), que emprega o termo prognóstico de enfermagem como indicador das condições que o cliente atingirá após o tratamento, incluindo aí a avaliação do processo de cuidar de si, medindo todas as suas fases. Esta avaliação baseiase no grau de dependência ou independência que o cliente terá após a assistência. $O$ prognóstico de enfermagem estima a probabilidade e a capacidade do paciente alcançar sua independência, em atender suas necessidades básicas, e orientar a assistência de enfermagem. ${ }^{(19,36)}$

DANIEL(19) emprega o termo avaliação para "fazer um levantamento ou verificação dos procedimentos de enfermagem utilizados e dos resultados obtidos no atendimento das necessidades básicas da pessoa humana". Propõe meios de avaliar o resultado, como por exemplo: observação sistematizada, estudo de curvas indicadoras, avaliação baseada na evolução de enf ermagem.

PAIM $^{(37)}$ define avaliação como uma linha de "feedback" que une continuamente as situações envolvidas no processo, enquanto CAMPEDELLI et al. ${ }^{(8)}$ apresentam a avaliação como resposta do pa- ciente às ações de enfermagem, propondo que essa metodologia deva ser centrada no auto-cuidado.

CIANCIARULLO ${ }^{(9)}$ afirma que a sistematização das atividades deve ser compatível com as necessidades do cliente, de sua família e da comunidade. A sistematização pode resultar num processo formal de avaliação da qualidade da assistência de enfermagem. Para que isto ocorra, é necessário exercer controle sobre o resultado das ações. A autora considera que o controle de qualidade da assistência visa assegurar a manutenção e a implementação da sua excelência.

Através do controle das componentes estruturais do processo de enfermagem, e dos resultados apresentados pelo usuário, é possível realizar as necessárias alterações e implementações para a melhoria da qualidade da assistência de enfermagem.

Pelo levantamento bibliográfico realizado neste estudo, pode-se perceber que a enfermagem brasileira, assim como a internacional, está dando os primeiros passos em busca de critérios ou elementos para subsidiar a avaliação dos resultados decorrentes da assistência.

Os termos encontrados neste estudo como $a v a$ liação de resultados estão expressos na Tabela 3 que demonstra o termo avaliação $(35,5 \%)$ como o mais empregado. Analisando-se os termos dessa tabela percebe-se que há relação entre os meios de avaliação de resultado proposto por DANIEL(19), como por exemplo: percepção como orientação casual intuitiva, opinião como observação intelectual orientada, critérios de avaliação como observação sistematizada, e monitorização como estudo de curvas e índices indicadores.

Tabela 3 - Terminologia identificada como AVALIAÇÃO em trabalhos referentes às atividades de enfermagem - 1988/1992. São Paulo, 1992.

\begin{tabular}{l|r|r}
\hline TERMO & \multicolumn{2}{c}{ F } \\
\hline Avaliaçăo & 5 & $35,5^{\star}$ \\
Percepçăo & 2 & 14,4 \\
Opiniăo & 2 & 14,4 \\
Objetivos & 2 & 14,4 \\
Critérios de avaliaçăo & 1 & 7,1 \\
Monitorizaçăo & 1 & 7,1 \\
Prognóstico de enfermagem & 1 & 7,1 \\
\hline TOTAL & 14 & 100,0 \\
\hline
\end{tabular}

" aproximados para efeito de cálculo

O termo prognóstico parece ter sido empregado a partir dos trabalhos de HORTA ${ }^{(27)}$, PAIM et al. ${ }^{(36) e}$ DANIEL(19). 
Observou-se também, que a utilização de alguns dos termos apresentados na Tabela 3, possuem relação com a avaliação enquanto resultado alcançado por uma determinada prescrição de enf ermagem.

Apesar da baixa freqüência de estudos identificados como avaliação de resultados da assistência, as áreas de especialidades que empregaram esta categoria foram as de saúde materno-infantil(4), saúde do adulto (7), saúde mental (1) e outras sem especificação (3).

Os dados acerca da avaliação dos resultados da assistência são incipientes, porém demonstram preocupação de alguns pesquisadores a respeito do "feed back" da assistência de enfermagem prestada.

\section{CONCLUSÃO}

A literatura revela que as atividades de enfermagem - diagnóstico, prescrição e avaliação - têm sido nomeadas com termos dif erentes, de significados semelhantes. Desse modo problemas, alterações, atividades ou manifestações do cliente são encontrados, nas referências analisadas, como diagnóstico; ações, atividades, condutas, orientação e intervenção como prescrição; e, percepção, opinião e prognóstico como avaliação do resultado da assistência de enfermagem.

A diversidade dos termos encontrados neste levantamento bibliográfico, sobre o uso de terminologia comum para as atividades universais de enfermagem, denota a gravidade do problema e revela a necessidade de estudos que possam aprofundar o assunto.

Em relação ao objetivo proposto, considera-se que há produção referente às atividades de enfermagem, e que os resultados podem reforçar a preocupação do ICN em padronizar linguagem universal para estas atividades.

Quanto à metodologia utilizada neste estudo, entende-se que foi apropriada para uma abordagem inicial do tema. Faz-se necessário, porém, apontar algumas limitações decorrentes, por exemplo, do breve período de tempo relacionado às publicações, e da utilização do acervo de uma determinada área geográfica, em detrimento de outras.

As limitações existentes, contudo, não comprometem a proposta deste trabalho, que é a de contribuir para a reflexão e discussão do tema em níveis nacional e internacional.

Os dados obtidos demonstram que a diversidade de linguagem sugere uma conf usão quanto aos referenciais teóricos, próprios para a enfermagem, comprometendo o desenvolvimento da profissão enquanto ciência e dificultando a construção de um corpo de conhecimentos.

É interessante notar que, no decorrer deste estudo, foram analisados alguns trabalhos em que se percebe haver uma tendência para a utilização do modelo proposto pela NANDA.

Diante do quadro traçado sobre as atividades de enfermagem no Brásil, com base na literatura especializada, recomenda-se que sejam realizados estudos mais amplos, com outras metodologias, de modo que se possa determinar a padronização da linguagem referente às atividades de enfermagem quer em âmbito nacional ou internacional.

\section{REFERÊNCIAS BIBLIOGRÁFICAS}

1. BENKO, M. A., CASTILHO, V. Operacionalização de um sitema de assistência de enf ermagem. In: CAMPEDELLI, M. C. et al. Processo de enfermagem na prática. São Paulo: Ática, 1989. cap. 7, p. 89-111.

2. BRASIL, Ministério da Saúde. Padrões minimos de assistência de enfermagem em recuperação da saúde - informe final. Brasilia, 1978 .

3. ___ Leis etc. Lei $n^{\circ} 7498$ de 25 de junho de 1986. Dispõe sobre a regulamentação do exercicio da enfermagem e dá outras providências. Diário Oficial da União, Brasilia, p. 9273-52 jun. 1986. Seção 1 ,

4. , Leis etc. Decreto $n^{\circ} 94406$ de 08 de junho de 1987. Regulamenta a Lei $n^{\circ} 7498$ de 25 de junho de 1986, que dispõe sobre o exercicio da enfermagem e de outras providências. Documenta, n.319, p. 157-60, 1987.

5. BULECHEK, G. M. et al. Defining and validating nursing intervention. N. Clin. N. Am., v. 27, n. 2, 1992.
6. CAMPEDELLI, M. C. Consulta de enf ermagem em geriatria: tópicos a serem abordados no exame físico - parte II. Rev. Esc. Enf. USP, v. 26, n. 1, p. 33-42, 1992.

$7 . \quad$ Consulta de enfermagem para o idoso: uma necessidade real. Rev. Paul. Enf., v. 10, n. 1, p. 32-37, 1991.

8. . et. al. Processo de enfermagem na prática. São Paulo, Atica, 1989.

9. CIANCIARULLO, T. I. Análise retrospectiva da qualidade de assistência de enfermagme em um hospital universitário. Tese (Livre-docència) - Escola de Enfermagem, Universidade de São Paulo, 1988. 243 p.

10. CLARK. J. L.ANG, N. Nursing's next advance: an international classilication for nursing practice. Int. Nurs. Rev. v. 39 , n. 4, p. 109-12, 1992. 
11. CRUZ, D. de A. .L. M. da, ARCURI, E. A. M. Diagnósticos de enfermagem dos pacientes internados com cardiopatia chagásica crônica. Rev. Esc. Enf. USP, v. 24, n.2, p. 26580, 1990.

12. . Diagnóstico de enfermagem e padrões de saude alternados em pacientes internados por cardiopatica chagásica crônica. (Mestrado) - Escola de Enfermagem, Universidade de São Paulo. 1989, 97p.

13. et al. Sistematização da assistência de enf ermagem em uma área de recuperação da saúde. Rev. Esc. Enf. USP, V. 21, N. ESP., P. 68-76, 1987.

14. CRUZ, I. C. F. da. Desenvolvimento histórico dos diagnósticos de enfermagem. Trabalho aprsentado na $3^{\circ}$ Semana Wanda Horta São Paulo, EEUSP, ago. 1992 (dat.)

15. Diagnóstico de enfermagem e sua aplicação: revisão da literatura. Rev. Esc. Enf. USP, v. 24, n. 1, p. 149-62, 1990.

16. Implementação do diganóstico de enfermagem na unidade de recuperação pós-anestésica - estudo piloto. Rev. Esc. Enf. USP, v. 24, n.3, p. 345-8, 1990.

17. Estudo descritivo sobre as caracteristicas definidoras do diagnóstico de enfermagem: disfunção sexual. Rev. Esc. Enf. USP, v. 26, n. 1, p. 43-64, 1992.

18. ; CRUZ, D. de A. L. M da. Os padrões de comportamento humano: uma proposta de tradução dos termos de Taxonomia I sobre diagnósticos de enfermagem. Rev. Esc. Enf. USP. v. 25, n. 1, p. 17-20, 1991.

19. DANIEL, L. F. A enfermagem plane jada. São Paulo, 1977.

20. FARIAS, S. N. et al. Diagnóstico de enfermagem: uma abordagem conceitual e prática. João Pessoa: Santa MArta, 1990.

21. FERRAZ, A. E. et al. Considerações sobre o uso dos termos: problemas, problema do paciente e problema de enfermagem. Rev. Esc. Enf. USP. v. 22, n. especial, p. 102-11, 1988.

22. GUTIERREZ, M. G. R. A intervenção do enfermeiro: uma análise a partir da prática. Tese (Doutorado) - Escola de Enf ermagem, Universidade de São Paulo. 1989, 101 p.

23. HORTA, W. de A. Diagnóstico de enfermagem: representação gra'fica. Enf. Novas Dimens., v. 3, n. 2, p. 75-7, 1977.

$24 . \quad$ A metodologia do processo de enfermagem. Rev. Bras. Enf., v. 24, n. 6, p.81-95, 1971.

$25 . \quad$ Considerações sobre o diagnóstico de enfermagem. Rev. Bras. Enf. v. 20, N. 1, P. 7-13, 1967.
26.

Diagnóstico de enfermagem: estudo básico de determinação da pdependência de enfermagem. Rev. Bras. Enf. v. 25, n. 4, p. 267-73, 1972.

27. Processo de enfermagem. São Paulo, E.P.U., 1979.

28. KELLY, M. A. Nursing diagnosis source book: guidelines for clinical application. Norwalk: Appleton - Century-Crafts, 1985.

29. KOLLER, E. M. P., MACHADO, H. B. Reflexões sobre a prática atual da enfermagem e prenúncios de mudanças para o século XXI. Rev. Bras. Enf., v. 45, n. 1, p. 74-9, jan. / mar. 1992.

30. MARIA, V. L. R. et al. Sistematização da assistência de enfermagem no Instituto "Dante Pazzanese" de cardiologia: relato de experiência. Rev. Esc. Enf. USP, n. 21, p. 77-87, 1987.

31. MARIA, V. L. R. Preparo de enfermeiras para a utilização de diagnósticos de enfermagem: relato de experiencia. Dissertação (Mestrado) - Escola de Enfermagem, Universidade de São Paulo. 1990, 136p.

32. Mc CLOSKEY, J. C., BULECHEK, G. M. Classification of nursing intervention. Iowa, 1992.

$33 . \quad$; BULECHEK, G. M. Nursing intervention classification (NIC), 1992.

34. NOBREGA, M. M. L da. Diagnósticos de enfermagem da NANDA e aTeoria das Necessidades Humanas de Horta. Dissertação (Mestrado) - Universidade Federal da Paraíba João Pessoa, 1991. 100p.

35. NURSING NEXT ADVANCE: development of an international classification for nursing practice. Final proposal submitted to the board of directors. Geneve, International Counsil of Nursing. 1991. 48p.

36. PAIM, L. et al: Iniciamento à metodologia do processo de enfermagem: uma abordagem centrada nas necessidades humanas básicas. Rio de Janeiro: Associação Brasileira de Enfermagem, 1973.

37. PAIM, L. Problemas, prescrições e planos: um estilo de assistência de enf ermagem. Cadernos Cientificos: ABEn, 1978.

38. PEREZ. M. L. de A. B. et al. Diagnóstico de enfermagem - um desafio de enfermagem para os anos 80. Rev. Bras. Enf., v. 43, n. 1, 2, 3, 4 p. 14-8, 1990.

39. TAXONOMY I Revised - 1990 with oflicial nursing diagnosis. St.Louis, North American Nursing Diagnosis Association. 1990.

Recebido para publicação em 28/11/93 